\title{
ncRNA2MetS: a manually curated database for non-coding RNAs associated with metabolic syndrome
}

\author{
Dengju Yao ${ }^{\text {Corresp., } 1,2,3}$, Xiaojuan Zhan ${ }^{4,5}$, Xiaorong Zhan ${ }^{6}$, Chee Keong Kwoh ${ }^{2}$, Yuezhongyi Sun ${ }^{1,5}$ \\ ${ }^{1}$ School of Software and Microelectronics, Harbin University of Science and Technology, Harbin, Heilongjiang, China \\ 2 School of Computer Science and Engineering, Nanyang Technological University, Singapore, Singapore \\ 3 College of Bioinformatics Science and Technology, Harbin Medical University, Harbin, Heilongjiang, China \\ ${ }^{4}$ College of Computer Science and Technology, Heilongjiang Institute of Technology, Harbin, Heilongjiang, China \\ 5 School of Computer Science and Technology, Harbin University of Science and Technology, Harbin, Heilongjiang, China \\ 6 Department of Endocrinology and Metabolism, the First Affiliated Hospital of Harbin Medical University, Harbin, Heilongjiang, China \\ Corresponding Author: Dengju Yao \\ Email address: ydkvictory@hrbust.edu.cn
}

Metabolic syndrome is a cluster of the most dangerous heart attack risk factors (diabetes and raised fasting plasma glucose, abdominal obesity, high cholesterol and high blood pressure), and has become a major global threat to human health. A number of studies have demonstrated that hundreds of non-coding RNAs, including miRNAs and IncRNAs, are involved in metabolic syndrome-related diseases such as obesity, type 2 diabetes mellitus, hypertension etc. However, these research results are distributed in a large number of literature, which is not conducive to analysis and use. There is an urgent need to integrate these relationship data between metabolic syndrome and non-coding RNA into a specialized database. To address this need, we developed a metabolic syndromeassociated non-coding RNA database (ncRNA2MetS) to curate the associations between metabolic syndrome and non-coding RNA. Currently, ncRNA2MetS contains 1068 associations between five metabolic syndrome traits and 627 non-coding RNAs (543 miRNAs and 84 IncRNAs) in four species. Each record in ncRNA2MetS database represents a pair of disease-miRNA (IncRNA) association consisting of non-coding RNA category, miRNA (IncRNA) name, name of metabolic syndrome trait, expressive patterns of noncoding RNA, method for validation, specie involved, a brief introduction to the association, the article referenced, etc. We also developed a user-friendly website so that users can access and download all data easily. In short, ncRNA2MetS is a complete and high-quality data resource for exploring the role of non-coding RNA in the pathogenesis of metabolic syndrome and seeking new treatment options. The website is freely available at http://www.biomed-bigdata.com:50020/index.html 
2 ncRNA2MetS: a manually curated database for non-

3 coding RNAs associated with metabolic syndrome

4

5

6

7

8

Dengju Yao ${ }^{1,2,3}$, Xiaojuan Zhan ${ }^{4,5}$, Xiaorong Zhan ${ }^{6}$, Chee Keong Kwoh ${ }^{2}$, Yuezhongyi Sun ${ }^{1,5}$

${ }^{1}$ School of Software and Microelectronics, Harbin University of Science and Technology, Harbin, Heilongjiang, China

${ }^{2}$ School of Computer Science and Engineering, Nanyang Technological University, Singapore

${ }^{3}$ College of Bioinformatics Science and Technology, Harbin Medical University, Harbin, Heilongjiang, China

${ }^{4}$ College of Computer Science and Technology, Heilongjiang Institute of Technology, Harbin, Heilongjiang, China

${ }^{5}$ School of Computer Science and Technology, Harbin University of Science and Technology, Harbin, Heilongjiang, China

${ }^{6}$ Department of Endocrinology and Metabolism, the First Affiliated Hospital of Harbin Medical University, Harbin, Heilongjiang, China

Corresponding Author:

Dengju Yao

Harbin, Heilongjiang, China

Email address: ydkvictory@hrbust.edu.cn

\section{Abstract}

Metabolic syndrome is a cluster of the most dangerous heart attack risk factors (diabetes and raised fasting plasma glucose, abdominal obesity, high cholesterol and high blood pressure), and has become a major global threat to human health. A number of studies have demonstrated that hundreds of non-coding RNAs, including miRNAs and lncRNAs, are involved in metabolic syndrome-related diseases such as obesity, type 2 diabetes mellitus, hypertension etc. However, these research results are distributed in a large number of literature, which is not conducive to analysis and use. There is an urgent need to integrate these relationship data between metabolic syndrome and non-coding RNA into a specialized database. To address this need, we developed a metabolic syndrome-associated non-coding RNA database (ncRNA2MetS) to curate the associations between metabolic syndrome and non-coding RNA. Currently, ncRNA2MetS contains 1068 associations between five metabolic syndrome traits and 627 non-coding RNAs (543 miRNAs and 84 lncRNAs) in four species. Each record in ncRNA2MetS database represents a pair of disease-miRNA (lncRNA) association consisting of non-coding RNA category, miRNA (lncRNA) name, name of metabolic syndrome trait, expressive patterns of 
40

41

42

43

44

45

46

\section{7}

48

49

50

51

52

53

54

55

56

57

58

59

60

61

62

63

64

65

66

67

68

69

70

71

72

73

74

75

76

77

78

79

non-coding RNA, method for validation, species involved, a brief introduction to the association, the article referenced, etc. We also developed a user-friendly website so that users can access and download all data easily. In short, ncRNA2MetS is a complete and high-quality data resource for exploring the role of non-coding RNA in the pathogenesis of metabolic syndrome and seeking new treatment options. The website is freely available at http://www.biomedbigdata.com:50020/index.html

\section{Introduction}

Metabolic syndrome (MetS) is a cluster of the most dangerous heart attack risk factors: diabetes and raised fasting plasma glucose, abdominal obesity, high cholesterol and high blood pressure (Alberti et al., 2005). It is estimated that around 20-25\% of the world's adult population have metabolic syndrome, making them three times more likely to have, and twice as likely to die from, a heart attack or stroke when compared to people without the syndrome (International Diabetes Federation, 2006). Metabolic syndrome has become a major threat to human health around the world. However, to date, the pathogenesis of metabolic syndrome continues to challenge experts. In recent years, a growing number of studies have suggested that many noncoding RNAs (ncRNAs), including small non-coding RNAs, particularly microRNAs (miRNAs), and long non-coding RNAs (lncRNAs), may be involved in metabolic syndrome-related diseases such as obesity, type 2 diabetes mellitus, hypertension etc. (Stoll et al., 2018; Sala et al., 2018; Esguerra et al., 2018; Cui et al, 2018; Lorente-Cebrián et al., 2019). Dysregulation of some miRNAs and lncRNAs disrupts the gene regulatory network, leading to metabolic syndrome and other related diseases. MiRNAs are $\sim 22 \mathrm{nt}$ non-coding small RNAs that negatively regulate gene expression at the post-transcriptional level (Bartel, 2004). Extensive research suggests that many miRNAs, such as miR-9 (Hu el al., 2018), miR-20b-5p (Katayama el al., 2019; Gentile et al., 2019), miR-802 (Kornfeld el al., 2013), let-7f (Gentile el al., 2019), miR-33 (Rayner el al., 2010), miR-375 (Sedgeman el al., 2019), and others are involved in glucose homeostasis, diabetes mellitus, abdominal obesity and cholesterol metabolism. Furthermore, some lncRNAs, a novel class of long non-coding RNA larger than 200nt, have been reported to be involved in the pathogenesis of type 2 diabetes mellitus and metabolic syndrome (Singer, Sussel, 2018; Losko, Kotlinowski, Jura, 2016; Wang et al., 2018).

Due to the important effects of metabolic syndrome on human health, it is urgent to develop a database dedicated to various biomarkers associated with metabolic syndrome, such as genes, miRNAs and lncRNAs. In recent years, several data resources and tools have been developed for storing metabolic disease-associated biomolecules, such as T-HOD, metabolicMine, PathCaseMAW, HMA and BioM2MetDisease. T-HOD (Dai et al., 2013) is a literature-based candidate gene database currently containing 837, 835 and 821 candidate genes for hypertension, obesity and diabetes, respectively. metabolicMine (Lyne et al., 2013) is a data warehouse with a specific focus on the genomics, genetics and proteomics of common metabolic diseases. PathCaseMAW (Cicek et al., 2013) provides a database-enabled framework and web-based computational tools for browsing, querying, analyzing and visualizing stored metabolic 
80

81

82

83

84

85

86

87

88

89

90

91

92

93

94

95

96

97

98

99

100

101

102

103

104

105

106

107

108

109

110

111

112

113

114

115

116

117 Data collection from literature in PubMed

networks. HMA (Pornputtapong, Nookaew, Nielsen, 2015) is a human metabolic atlas website which provides information about human metabolism. These four software resources described above have provided important support for the study of the pathogenesis of metabolic diseases. However, they do not contain non-coding RNA information related to metabolic diseases. BioM2MetDisease (Xu et al., 2017) is a manually curated database containing 2681 entries of associations between 1147 biomolecules and 78 metabolic diseases. Though it is a very useful tool for studying metabolic diseases, BioM2MetDisease is not a database dedicated to metabolic syndrome. It contains miRNAs associated with 78 metabolic diseases but does not include hypertension and hypo-HDL cholesterolemia, which are two important traits of metabolic syndrome. In addition, it does not contain IncRNAs associated with metabolic syndrome and miRNAs from the last two years.

In addition to the five data resources described above, there are other resources and tools for studying human diseases. miR2Disease (Jiang et al., 2009) is a manually curated database which contains 1939 curated relationships between 299 human miRNAs and 94 human diseases. phenomiR (Ruepp, Kowarsch, Theis, 2012) provides miRNA and target relations from these studies on the association of dysregulated miRNAs and diseases. HMDB (v3.0) (Wishart et al., 2012) is a resource dedicated to the human metabolome, which includes more than 40,000 annotated metabolite entries. HMDD (v3.0) (Huang et al., 2018) manually collects a significant number of miRNA-disease association entries. All these databases have provided valuable tools for exploring the roles of these biomolecules in human diseases, but they are not designed specifically for metabolic syndrome. When faced with so many data resource options, it is difficult to find the desired data for doctors who focus on metabolic syndrome. In addition, more and more non-coding RNAs associated with metabolic syndrome have been discovered in the last two years (Saeedi et al., 2019; Zhang et al., 2019; Zhang et al., 2018; Smieszek et al., 2019; Lin et al., 2019).

So far, there is still no non-coding RNA database dedicated to metabolic syndrome. There is an urgent need for a specialized data resource containing all the latest non-coding RNAs associated with metabolic syndrome. To meet this demand, we have developed the ncRNA2MetS database which contains the latest and most complete MetS-miRNA (lncRNA) associations validated by various biological experiments. We carefully reviewed 571 articles about relationship between various metabolic syndrome traits and non-coding RNA and gained 1068 associations between five metabolic syndrome traits and 627 non-coding RNAs (543 miRNAs and 84 lncRNAs) in four species. We hope that this database can help doctors specializing in metabolic syndrome to explore the pathogenesis and treatments of metabolic syndrome.

\section{Materials \& Methods}


118

119

120

121

122

123

124

125

126

127

128

129

130

131

132

133

134

135

136

137

138

139

140

141

142

143

144

145

146

147

148

149

150

151

152

153

154

155

156

157

According to the International Diabetes Federation (IDF) definition, a person with metabolic syndrome must have central obesity (defined as waist circumference with ethnicity-specific values) plus any two of the following four factors: (1) raised triglycerides, or specific treatment for this lipid abnormality; (2) reduced HDL cholesterol, or specific treatment for this lipid abnormality; (3) raised blood pressure, or treatment of previously diagnosed hypertension; (4) raised fasting plasma glucose, or previously diagnosed type 2 diabetes (Alberti et al., 2005; International Diabetes Federation, 2006). Therefore, we divided the risk factors of metabolic syndrome into five traits: central obesity, type 2 diabetes mellitus, hypertension, hypertriglyceridemia and hypo-HDL cholesterolemia.

Referring to Xu's method (Xu et al., 2017), we manually collected and curated MetS-miRNA (lncRNA) associations from related articles in the PubMed database. First, we used 'non-coding RNA', 'ncRNA', 'microRNA', 'miRNA', 'long non-coding RNA', 'lncRNA' and each metabolic syndrome trait as search terms to search the PubMed database by Title/Abstract retrieval method. As a result, we gained more than 3000 related articles published since 2007. We filtered out a large number of irrelevant articles or reviews by reading abstracts and finally selected 571 articles that were really focused on association between metabolic syndrome trait and miRNA (lncRNA). Then, we manually extracted MetS-miRNA (lncRNA) associations by reading these selected articles in detail. In the process of extracting these associations, the detailed information about MetS-miRNA (lncRNA) association were collected, including noncoding RNA category, miRNA (lncRNA) name, name of metabolic syndrome trait, ICD-11 classification and DO (Disease Ontology) identifier for metabolic syndrome trait, method for validation (e.g. RNA-seq, luciferase report assays, gene knock-out), detected tissue (e.g. serum, adipose tissue, liver), expressive patterns (e.g. up-regulation, down-regulation, differential expression), name of the gene regulated by miRNA (lncRNA), species involved (e.g. homo sapiens, mus musculus, rattus norvegicus), referenced article (PubMed ID, title, year of publication) and a brief introduction to this association in the referenced article. Following previous research rules (Jiang et al., 2009; Xu et al., 2017; Huang et al., 2018), we only collected MetS-miRNA (lncRNA) associations validated by various biological experiments in this process. At the same time, in order to ensure the authenticity and reliability of the extracted information, each MetS-miRNA (IncRNA) association was confirmed by at least two scholars. Finally, in order to ensure consistency with other data resources, we standardized the names of miRNAs, lncRNAs and metabolic syndrome traits. We provided miRBase (Kozomara, Birgaoanu, Griffiths-Jones, 2019) identifier for miRNAs, NONCODE (Fang et al., 2017) identifier for lncRNAs, ICD-11 classification and DO identifier for metabolic syndrome trait. The process of constructing the ncRNA2MetS database is shown in Figure 1.

\section{Database and website development}

In order to facilitate users to access and use the data in the ncRNA2MetS database, we developed a user-friendly website providing data browsing, searching and downloading function. The website was implemented in Java programing language, and all data were stored in MySQL database. The website is freely available at http://www.biomed-bigdata.com:50020/index.html 


\section{Results}

160

161

162

163

164

165

166

167

168

169

170

171

172

173

174

175

176

177

178

179

180

181

182

183

184

185

186

187

188

189

190

191

192

193

194

195

196

\section{Database contents}

By April 2019, we gained 3699 potential articles from PubMed using "Title/Abstract" searching. After manual screening according to the relevance of the research contents, 571 articles were selected for reading in detail, and 1068 MetS-miRNA (lncRNA) associations were identified finally. To describe MetS-miRNA (lncRNA) association in more detail, each record about MetSmiRNA (lncRNA) association in ncRNA2MetS database consists of non-coding RNA category, miRNA (lncRNA) name, miRBase identifier for miRNA, NONCODE identifier for lncRNA, name of metabolic syndrome trait, ICD-11 classification and DO identifier for metabolic syndrome trait, method for validation, detected tissue, expressive patterns, name of the gene regulated by miRNA (IncRNA), species involved, information of cited articles and a brief introduction to the associations in this referenced article. (see Materials and Methods). Currently, the ncRNA2MetS database contains 1068 associations between five metabolic syndrome traits (central obesity, type 2 diabetes mellitus, hypertension, hyperlipidemia and hypo-HDL cholesterolemia) and 627 non-coding RNAs (543 miRNAs and 84 lncRNAs) in four species (homo sapiens, mus musculus, rattus norvegicus and Sus scrofa). Among the 1068 associations, the number of miRNAs related to central obesity, type 2 diabetes mellitus, hypertension, hyperlipidemia and hypo-HDL cholesterolemia are 288, 207, 96, 50 and 41, respectively. In addition, the number of miRNAs reported to be related to metabolic syndrome is 36 (Figure 2a). The number of IncRNAs related to central obesity, type 2 diabetes mellitus, hypertension, hyperlipidemia, hypo-HDL cholesterolemia and metabolic syndrome are 42, 28, 12, 1, 2 and 3, respectively (Figure 2b).

\section{Database interface}

ncRNA2MetS database implements a user-friendly website interface through which users can access all data in ncRNA2MetS conveniently and easily. The website consists of six parts, namely HOME, BROWSE, SEARCH, DOWNLOAD, SUBMIT and HELP. The 'HOME' page shows a brief introduction about metabolic syndrome, miRNA and lncRNA while 'BROWSE' page (Figure 3a) and 'SEARCH' page (Figure 3b) provide data query. On the 'BROWSE' page, users can click a specific miRNA, IncRNA or metabolic syndrome trait to browse the MetSncRNA associations. Then, the website will return all MetS-ncRNA associations that meet the query criteria. If too many association entries are returned, users can specify ncRNA category, species or validation method to screen for required entries. For example, if users specify 'homo sapiens' as species, the website will return all associations related to 'homo sapiens' (Figure 3c). The 'SEARCH' page provides users with faster and more accurate query method, which support 'Accurate Search' and 'Fuzzy Search'. For 'Accurate Search', users can input an accurate miRNA (lncRNA) name or metabolic syndrome trait name, or both, then click 'Search' button to query the required associations. On the contrary, for 'Fuzzy Search', users only need to input partial names of a miRNA (lncRNA) or metabolic syndrome trait to query the required 
197 relationships. It should be noted that query keywords are case-insensitive. In addition, users can

198

199

200

201

202

203

204

205

206

207

208

209

210

211

\section{Examples of using ncRNA2MetS}

213

214

215

216

217

218

219

220

221

222

223

224

225

226

227

228

229

230

231

232

233

234

235

236 'HELP' page or contact us via e-mail.

specify 'Validation Methods' to narrow query scope. For example, if the user specifies 'qRTPCR' as the validation method, the website will return all associations that match query keywords and have been validated by qRT-PCT. Similar to 'BROWSE', in the search result page, users can also filter MetS-ncRNA associations by selecting specified ncRNA category, species or validation method. Finally, users can browse the detailed information about a specific MetS-ncRNA association by clicking a hyperlink to the 'Details' page (Figure 3d).

In addition to freely querying MetS-ncRNA associations stored in ncRNA2MetS, users can also submit novelty associations validated by their own experiments. They can do this on the 'SUBMIT' page (Figure 3e) and must provide detailed information about the new association. Our committee will regularly review new submissions. Once the submitted association is confirmed, it will be added into ncRNA2MetS. Furthermore, users can freely and easily download all MetS-ncRNA associations in the 'DOWNLOAD' page. Finally, if users encounter any difficulties or problems in using the ncRNA2MetS, they can find help information on

In this section, we will use examples to show you how to use the ncRNA2MetS database. First, users can input 'miR-155-5p' as the miRNA name on the 'SEARCH' page and then click the 'Search' button. A result page will be returned and will display all records about miR-155-5p, including those of different species and various metabolic syndrome traits. On the result page, one can easily notice that miR-155-5p is related to all five metabolic syndrome traits, which implies that it has a very important impact on metabolic syndrome. In fact, miR-155-5p has been reported to be a risk factor of metabolic syndrome. ncRNA2MetS also supports searching by the name of metabolic syndrome trait, and this can facilitate the study of pathogenesis for a certain specific metabolic syndrome trait. For example, users can input 'obesity' as the name of metabolic syndrome trait on the 'SEARCH' page and will find that a number of miRNAs and lncRNAs such as miR-21, miR-155-5p and Parall showed abnormal expression in human obesity. The introduction to these associations in ncRNA2MetS shows that a reduced level of miR-21 might be associated with obesity and its related metabolic traits such as hyperinsulinemia (Ghorbani et al., 2018); Obese subjects have increased expressions of miR155-5p and miR-122, two miRNAs related to inflammation and iron metabolism, respectively, at both the systemic and sperm levels (López et al., 2018); Furthermore, a novel component of the adipogenic transcriptional regulatory network defining the lncRNA Parall is identified as an obesity-sensitive regulator of adipocyte differentiation and function (Firmin, et al., 2017).

As a feature, ncRNA2MetS also supports querying lncRNAs associated with metabolic syndrome. For example, by inputting 'H19', the result page will show all records of relationships between ' $\mathrm{H} 19$ ' and various metabolic syndrome traits including obesity, type 2 diabetes mellitus and hypo-HDL cholesterolemia in three species. The functional description in ncRNA2MetS shows that imprinted lncRNA H19 increases upon cold-activation and decreases in obesity in BAT (Schmidt et al., 2018); Related studies reveal a previously undescribed double-negative

Peer) reviewing PDF | (2019:06:38652:2:0:NEW 10 Sep 2019) 
237 feedback loop between sponge lncRNA and target miRNA that contributes to glucose regulation

238

239

240

241

242

243

244

245

246

247

248

249

250

251

252

253

254

255

256

257

258

259

260

261

262

263

264

265

266

267

268

269

270

271

272

273

274

275

in muscle cells (Gao et al., 2014); A H19-miR130b pathway regulating lipid metabolism and inflammation response in ox-LDL-treated Raw264.7 cells provides new targets for atherosclerosis treatment (Han et al., 2018). Overall, ncRNA2MetS can be used as a highquality and most complete data resource for studying the roles of miRNAs and lncRNAs involved in metabolic syndrome.

\section{Database analysis}

Currently, ncRNA2MetS provides almost all the research results related to the association between metabolic syndrome and non-coding RNA. Comprehensive analysis of the data in ncRNA2MetS can help people better explore the relationship between metabolic syndrome and non-coding RNA. For this purpose, a relational network between metabolic syndrome traits and ncRNAs (miRNAs and lncRNAs) is constructed using Cytoscape software (Figure 4). In the MetS-ncRNA association network, nodes represent metabolic syndrome traits and ncRNAs, and edges represent the relationships between them. For miRNAs and lncRNAs, degree represents the number of associated metabolic syndrome traits, and also indicates their importance for researching the pathogenesis and treatment of metabolic syndrome. Fig. 4 shows that the degrees of miR-122, miR-155-5p and miR-146a-5p (green node) were largest among the numerous miRNAs, which are related to all five metabolic syndrome traits (obesity, type 2 diabetes mellitus, hypertension, hyperlipidemia and hypo-HDL cholesterolemia), or have been reportedly involved in metabolic syndrome. This result implies that these three miRNAs play an important role in the study of metabolic syndrome. In addition, the lncRNA with the highest degree is H19, which is related to obesity, type 2 diabetes mellitus, and hypo-HDL cholesterolemia, and is reported to be involved in the pathogenesis of metabolic syndrome. Further and deeper analysis of the ncRNA2MetS data will yield more interesting results.

\section{Discussion}

Metabolic syndrome has become one of the most important diseases threatening human health worldwide. There is increasing evidence suggesting that metabolic syndrome is associated with abnormal expression of some ncRNAs, including miRNAs and lncRNAs. A database dedicated to metabolic syndrome-ncRNA association is helpful in studying the pathogenesis and treatments of metabolic syndrome. For this purpose, we developed ncRNA2MetS, a database containing almost all experimentally supported metabolic syndrome-ncRNA associations. Currently, ncRNA2MetS contains 1068 validated associations between five metabolic syndrome traits and 627 ncRNAs (543 miRNAs and 84 lncRNAs) in four species.

In recent years, some researchers have developed several high-quality databases, such as BioM2MetDisease (Xu et al., 2017) and HMDD (Huang et al., 2018), to provide metabolic disease-miRNAs associations. Nevertheless, these databases are not dedicated to metabolic syndrome and do not cover all the metabolic syndrome traits. For example, BioM2MetDisease contains 2681 entries of relationships between 524 miRNAs and 45 metabolic diseases across 14 
276 species. This is a database with very rich storage content, but it is not specifically for metabolic 277 syndrome. Though BioM2MetDisease contains a large number of miRNAs associated with 278 obesity, type 2 diabetes mellitus and dyslipidemia, it lacks miRNA information related to 279 hypertension and hypo-HDL cholesterolemia. Furthermore, although a large number of new 280 miRNAs and lncRNAs related to metabolic syndrome have been identified and reported in the 281 past two years, BioM2MetDisease has not been updated with the latest findings. HMDD (v3.0) is

282

283

284

285

286

287

288

289

290

291

292

293

294

295

296

297

298

299

300

301

302

303

304

305

306

307

308

309

310

311

312

313

314

a database that curates experiment-supported evidence for human miRNA and disease associations. Currently, HMDD contains 32281 miRNA-disease association entries which include 1102 miRNA genes and 850 diseases. Similar to BioM2MetDisease, HMDD is not specifically for metabolic syndrome, and it does not contain miRNAs associated with hypo-HDL cholesterolemia. Furthermore, the number of miRNAs contained in HMDD is far less than ncRNA2MetS. To demonstrate the value of ncRNA2MetS, we comprehensively compared the amount of non-coding RNAs associated with various metabolic syndrome traits contained in BioM2MetDisease, HMDD and ncRNA2MetS. The results showed that ncRNA2MetS contained significantly more miRNAs associated with metabolic syndrome than both BioM2MetDisease and HMDD (Figure 5a). More concretely, the number of miRNAs associated with obesity, type 2 diabetes mellitus, hypertension, hypertriglyceridemia, hypo-HDL cholesterolemia and metabolic syndrome were 225, 204, 0, 24, 0 and 39 in BioM2MetDisease; 80, 128, 65, 8, 0 and 38 in HMDD; and 288, 207, 96, 50, 41 and 36 in ncRNA2MetS, respectively. Finally, ncRNA2MetS provides not only miRNAs but also lncRNAs associated with metabolic syndrome, and covers the latest research findings up to April 2019.

The prevalence of metabolic syndrome, including central obesity, type 2 diabetes mellitus, hypertension, hypertriglyceridemia and hypo-HDL cholesterolemia, is growing globally, and the amount of studies on metabolic syndrome is also increasing rapidly. To illustrate the research trend of ncRNAs related to metabolic syndrome, we counted the number of articles about noncoding RNA studies related to metabolic syndrome between 2007 and April 2019 (Figure 5b). Clearly, the amount of research about the association between ncRNAs and metabolic syndrome has increased rapidly in the past two years. Therefore, it will become a trend that more and more metabolic syndrome-ncRNA associations will be identified and validated in the future. We will keep track of the latest advances in the study of relationship between metabolic syndrome and non-coding RNA, and update ncRNA2MetS database regularly. In addition, we will focus on more types of non-coding RNA such as circular RNA, snoRNA, etc. and add associations between metabolic syndrome and these ncRNAs into ncRNA2MetS to increase its coverage. Furthermore, we will develop more powerful data analysis tools such as network visualization tool to help researchers better study the pathogenesis and treatment of metabolic syndrome in the future. In a word, we hope that ncRNA2MetS can be used as an effective tool for studying the mechanism of non-coding RNAs in metabolic syndrome.

14 Conclusions 
315 A growing number of studies have suggested that many non-coding RNAs, including miRNAs 316 and lncRNA, are involved in metabolic syndrome and its traits. In this article, we introduced 317 ncRNA2MetS, a user-friendly web-based tool developed for curating the association between 318 metabolic syndrome and ncRNAs (miRNA and lncRNAs). ncRNA2MetS currently contains 3191068 associations between five metabolic syndrome traits and 627 ncRNAs (543 miRNAs and 84 lncRNAs) in four species. ncRNA2MetS has covered almost all relevant researches about the association between metabolic syndrome and ncRNAs between 2007 and 2019. It is expected that ncRNA2MetS will serve as a valuable data resource that will help researchers better study the pathogenesis and treatments of metabolic syndrome.

\section{References}

Alberti KGMM, Zimmet P, Shaw J. 2005. The metabolic syndrome--a new worldwide definition. The Lancet 366(9491):1059-1062 DOI 10.1016/S0140-6736(05)67402-8

Bartel DP. 2004. MicroRNAs: genomics, biogenesis, mechanism, and function. Cell 116(2): 281-297 DOI 10.1016/S0092-8674(04)00045-5.

331 Cicek A E, Qi XJ, Cakmak A, Johnson SR, Han X, Alshalwi S, Ozsoyoglu ZM, Ozsoyoglu G. 2013. An online system for metabolic network analysis. Database: The Journal of Biological Databases and Curation 2014 DOI 10.1093/database/bau091. 2018. The long non-coding RNA Gm10768 activates hepatic gluconeogenesis by sequestering microRNA-214 in mice. The Journal of Biological Chemistry 293(11): 4097-4109 DOI 10.1074/jbc.M117.812818.

Dai HJ, Wu JCY, Tsai RTH, Pan WH, Hsu WL. 2013. T-HOD: a literature-based candidate gene database for hypertension, obesity and diabetes. Database: The Journal of Biological Databases and Curation 2013 DOI 10.1093/database/bas061.

Esguerra JLS, Nagao M, Ofori JK, Wendt A, Eliasson L. 2018. MicroRNAs in islet hormone secretion. Diabetes, Obesity and Metabolism 20(S2): 11-19 DOI 10.1111/dom.13382.

342 Fang SS, Zhang LL, Guo JC, Niu YW, Wu Y, Li H, Zhao LH, Li XY, Teng XY, Sun XH, 343 Sun L, Zhang MQ, Chen RS, Zhao Y. 2017. NONCODE V5: a comprehensive annotation 344 database for long non-coding RNAs. Nucleic Acids Research 46(D1): D308-D314 DOI 10.1093/nar/gkx1107.

Firmin FF, Oger F, Gheeraert C, Dubois-Chevalier J, Vercoutter-Edouart AS, Alzaid F, Mazuy C, Dehondt H, Alexandre J, Derudas B, Dhalluin Q, Ploton M, Berthier A, The RBM14/CoAA-interacting, long intergenic non-coding RNA Parall regulates adipogenesis and coactivates the nuclear receptor PPAR $\gamma$. Scientific Reports 7(1): 14087 DOI 10.1038/s41598-017-14570-y. 
355

356

357

358

359

360

361

362

363

364

365

366

367

368

369

370

371

372

373

374

375

376

377

378

379

380

381

382

383

384

385

386

387

388

389

390

391

392

393

394

metabolism in muscle cells. Nucleic Acids Research 42(22): 13799-13811 DOI

\subsection{3/nar/gku1160.}

Gentile AM, Lhamyani S, Coín-Aragüez L, Clemente-Postigo M, Olivera WO, RomeroZerbo SY, García-Serrano S, García-Escobar E, Zayed H, Doblado E, Bermúdez-Silva FJ, Murri M, Tinahones FJ, Bekay RE. 2019. miR - 20b, miR - 296, and Let - 7f Expression in Human Adipose Tissue is Related to Obesity and Type 2 Diabetes. Obesity 27(2): 245-254 DOI 10.1002/oby.22363.

\section{Ghorbani S, Mahdavi R, Alipoor B, Panahi G, Esfahani EN, Razi F, Taghikhani M,} Meshkani R. 2018. Decreased serum microRNA-21 level is associated with obesity in healthy and type 2 diabetic subjects. Archives of Physiology Biochemistry 124(4): 300-305 DOI 10.1080/13813455.2017.1396349.

Han Y, Ma J, Wang JP, Wang LP. 2018. Silencing of H19 inhibits the adipogenesis and inflammation response in ox-LDL-treated Raw264. 7 cells by up-regulating miR-130b. Molecular Immunology 93: 107-114 DOI 10.1016/j.molimm.2017.11.017.

Hu DZ, Wang Y, Zhang HY, Kong DL. 2018. Identification of miR-9 as a negative factor of insulin secretion from beta cells. Journal of Physiology and Biochemistry 74(2): 291-299 DOI 10.1007/s13105-018-0615-3.

Huang Z, Shi JC, Gao YX, Cui CM, Zhang S, Li JW, Zhou Y, Cui QH. 2018. HMDD v3. 0: a database for experimentally supported human microRNA-disease associations. Nucleic Acids Research 47(D1): D1013-D1017 DOI 10.1093/nar/gky1010.

International Diabetes Federation. 2006. The IDF consensus worldwide definition of the metabolic syndrome. Brussels: International Diabetes Federation. 2006: 4

Jiang QH, Wang YD, Hao YY, Juan LR, Teng MX, Zhang XJ, Li MM, Wang GH, Liu YL. 2009. miR2Disease: a manually curated database for microRNA deregulation in human disease. Nucleic Acids Research 37(suppl_1): D98-D104 DOI 10.1093/nar/gkn714.

Katayama M, Wiklander OPB, Fritz T, Caidahl K, EI-Andaloussi S, Zierath JR, Krook A. 2019. Circulating Exosomal miR-20b-5p Is Elevated in Type 2 Diabetes and Could Impair Insulin Action in Human Skeletal Muscle. Diabetes 68(3): 515-526 DOI 10.2337/db18-0470. Kornfeld JW, Baitzel C, Könner AC, Nicholls HT, Vogt MC, Herrmanns K, Scheja L, Haumaitre C, Wolf AM, Knippschild U, Seibler J, Cereghini S, Heeren J, Stoffel M, Brüning JC. 2013. Obesity-induced overexpression of miR-802 impairs glucose metabolism through silencing of Hnflb. Nature 494: 111-115 DOI 10.1038/nature11793.

Kozomara A, Birgaoanu M, Griffiths-Jones S. 2019. miRBase: from microRNA sequences to function. Nucleic Acids Research 47(D1): D155-D162 DOI 10.1093/nar/gky1141.

La Sala L, Micheloni S, De Nigris V, Prattichizzo F, Ceriello A. 2018. Novel insights into the regulation of miRNA transcriptional control: implications for T2D and related complications. Acta Diabetologica 55(10): 989-998 DOI 10.1007/s00592-018-1149-4.

López P, Castro A, Flórez M, Miranda K, Aranda P, Sánchez-González C, Llopis J, Arredondo M. 2018. miR-155 and miR-122 expression of spermatozoa in obese subjects. Frontiers in Genetics 9: 175 DOI 10.3389/fgene.2018.00175. 
395

396

397

398

399

400

401

402

403

404

405

406

407

408

409

410

411

412

413

414

415

416

417

418

419

420

421

422

423

424

425

426

427

428

429

430

431

432

433

434

Lorente-Cebrián S, González-Muniesa P, Milagro FI, Martínez JA. 2019. MicroRNAs and other non-coding RNAs in adipose tissue and obesity: emerging roles as biomarkers and therapeutic targets. Clinical Science 133(1): 23-40 DOI 10.1042/CS20180890.

Losko M, Kotlinowski J, Jura J. 2016. Long noncoding RNAs in metabolic syndrome related disorders. Mediators of Inflammation 2016: 1-12 DOI 10.1155/2016/5365209c.

Lyne M, Smith RN, Lyne R, Aleksic J, Hu FY, Kalderimis A, Stepan R, Micklem G. 2013. metabolicMine: an integrated genomics, genetics and proteomics data warehouse for common metabolic disease research. Database: The Journal of Biological Databases and Curation 2013 DOI 10.1093/database/bat060.

Pornputtapong N, Nookaew I, Nielsen J. 2015. Human metabolic atlas: an online resource for human metabolism. Database: The Journal of Biological Databases and Curation 2015 DOI 10.1093/database/bav068.

Rayner KJ, Suárez Y, Dávalos A, Parathath S, Fitzgerald ML, Tamehiro N, Fisher EA, Moore KJ, Fernández-Hernando C. 2010. MiR-33 contributes to the regulation of cholesterol homeostasis. Science 328(5985): 1570-1573 DOI 10.1126/science.1189862.

Ruepp A, Kowarsch A, Theis F. 2012. PhenomiR: MicroRNAs in human diseases and biological processes. Next-Generation MicroRNA Expression Profiling Technology. Humana Press, 2012: 249-260 DOI 10.1007/978-1-61779-427-8_17.

Saeedi Borujeni MJ, Esfandiary E, Baradaran A, Valiani A, Ghanadian M, CodoñerFranch P, Basirat R, Alonso-Iglesias E, Mirzaei H, Yazdani A. 2019. Molecular aspects of pancreatic $\beta$-cell dysfunction: Oxidative stress, microRNA, and long noncoding RNA. Journal of Cellular Physiology 234(6): 8411-8425 DOI 10.1002/jcp.27755.

Schmidt E, Dhaouadi I, Gaziano I, Oliverio M, Klemm P, Awazawa M, Mitterer G, Fernandez-Rebollo E, Pradas-Juni M, Wagner W, Hammerschmidt P, Loureiro R, Kiefer C, Hansmeier NR, Khani S, Bergami M, Heine M, Ntini E, Frommolt P, Zentis P, Ørom UA, Heeren J, Blüher M, Bilban M, Kornfeld JW. 2018. LincRNA H19 protects from dietary obesity by constraining expression of monoallelic genes in brown fat. Nature Communications. 9(1): 3622 DOI 10.1038/s41467-018-05933-8.

Sedgeman LR, Beysen C, Solano MAR, Michell DL, Sheng QH, Zhao SL, Turner S, Linton MF, Vickers KC. 2019. Beta cell secretion of miR-375 to HDL is inversely associated with insulin secretion. Scientific Reports 9(1): 3803 DOI 10.1038/s41598-019-40338-7.

Singer RA, Sussel L. 2018. Islet long noncoding RNAs: a playbook for discovery and characterization. Diabetes 67(8): 1461-1470 DOI 10.2337/dbi18-0001.

Smieszek A, Kornicka K, Szlapka-Kosarzewska J, Androvic P, Valihrach L, Langerova L, Rohlova E, Kubista M, Marycz K. 2019. Metformin Increases Proliferative Activity and Viability of Multipotent Stromal Stem Cells Isolated from Adipose Tissue Derived from Horses with Equine Metabolic Syndrome. Cells 8(2): 80 DOI 10.3390/cells8020080.

Stoll L, Sobel J, Rodriguez-Trejo A, Guay C, Lee KL, Venø MT, Kjems J, Laybutt DR, Regazzi R. 2018. Circular RNAs as novel regulators of $\beta$-cell functions in normal and disease conditions. Molecular Metabolism 9: 69-83 DOI 10.1016/j.molmet.2018.01.010. 
435 Wang JP, Yang WL, Chen ZZ, Chen J, Meng YH, Feng BQ, Sun LB, Dou L, Li J, Cui QH, 436 Yang JC. 2018. Long noncoding RNA lncSHGL recruits hnRNPA1 to suppress hepatic 437 gluconeogenesis and lipogenesis. Diabetes 67(4): 581-593 DOI 10.2337/db17-0799.

438 Wishart DS, Jewison T, Guo AC, Wilson M, Knox C, Liu YF, Djoumbou Y, Mandal R, 439 Aziat F, Dong E, Bouatra S, Sinelnikov I, Arndt D, Xia JG, Liu P, Yallou F, Bjorndahl T, 440 Perez-Pineiro R, Eisner R, Allen F, Neveu V, Greiner R, Scalbert A. 2013. HMDB 3.0- the 441 human metabolome database in 2013. Nucleic Acids Research 41(D1): D801-D807 DOI

442 10.1093/nar/gks1065.

443 Li XH, Tang SJ, Gui WW, Erik M, Tao TT, Li L, Wu F, Zhou JQ, Zheng FP, Li H. 2019.

444 Circulating miR-143-3p inhibition protects against insulin resistance in Metabolic Syndrome via 445 targeting of the insulin-like growth factor 2 receptor. Translational Research 205: 33-43 DOI

$446 \quad 10.1016 / j \cdot \operatorname{trs} 1.2018 .09 .006$

447 Xu YJ, Yang HX, Wu T, Dong Q, Sun ZG, Shang DS, Li F, Xu YQ, Su F, Liu SY, Zhang

448 YP, Li X. 2017. BioM2MetDisease: a manually curated database for associations between 449 microRNAs, metabolites, small molecules and metabolic diseases. Database: The Journal of

450 Biological Databases and Curation 2017 DOI 10.1093/database/bax037.

451 Zhang BH, Shen CA, Zhu BW, An HY, Zheng B, Xu SB, Sun JC, Sun PC, Zhang W, Wang 452 J, Liu JY, Fan YQ. 2019. Insight into miRNAs related with glucometabolic disorder.

453 Biomedicine \& Pharmacotherapy 111: 657-665 DOI 10.1016/j.biopha.2018.12.123.

454 Zhang X, Li DY, Reilly MP. 2019. Long intergenic noncoding RNAs in cardiovascular

455 diseases: Challenges and strategies for physiological studies and translation. Atherosclerosis 281:

456 180-188 DOI 10.1016/j.atherosclerosis.2018.09.040. 
Figure 1

The flowchart of the ncRNA2MetS database design

The whole process is divided into three stages: (A) Literature retrieval; (B) Data extraction; (C) Database and website development.

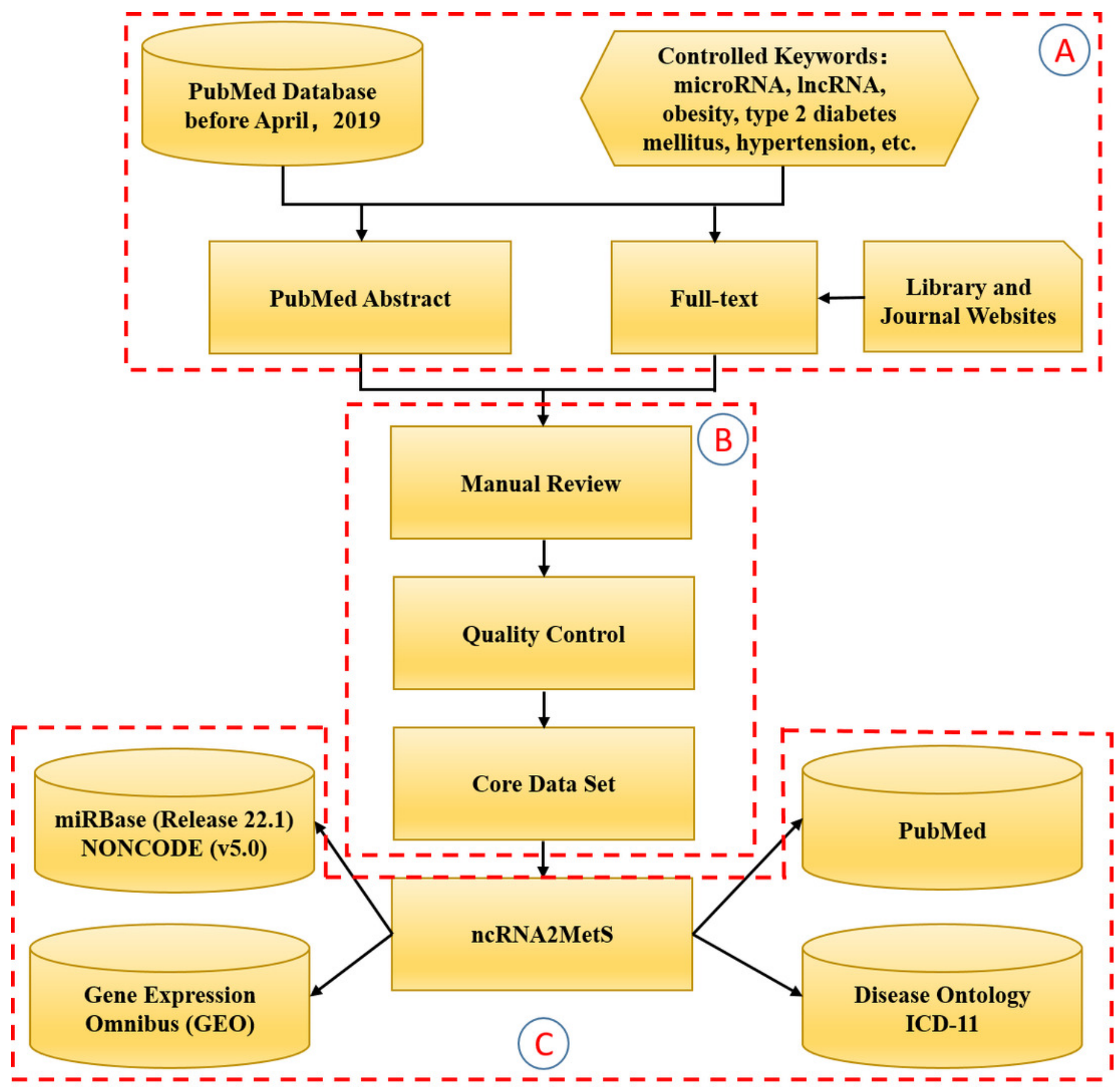




\section{Figure 2}

The statistics of ncRNAs contained in the ncRNA2MetS database

(A) The distribution of miRNAs in various metabolic syndrome traits. (B) The distribution of IncRNAs in various metabolic syndrome traits. (C) The distribution of miRNAs in different species. (D) The distribution of IncRNAs in different species.

The distribution of microRNAs in various metabolic syndrome traits

(A)

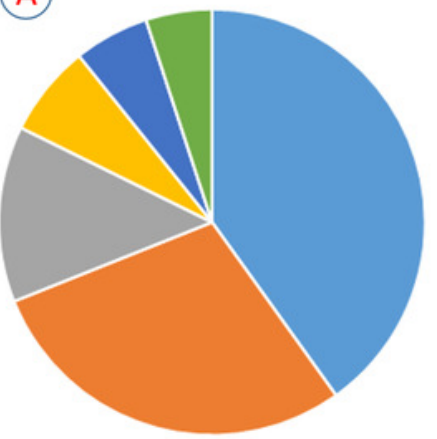

- Obesity

- Type II Diabetes Mellitus

- Hyperten sion

- Hyper lipidemia

- hypo-HDL cholesterolemia

- Met abolic Syndrome

The distribution of microRNAs in different species

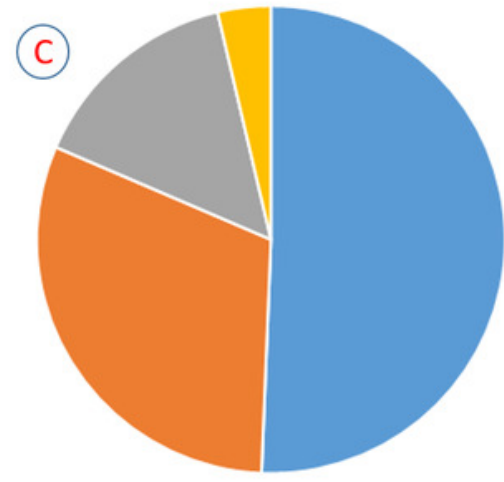

The distribution of IncNRAs in various metabolic syndrome traits

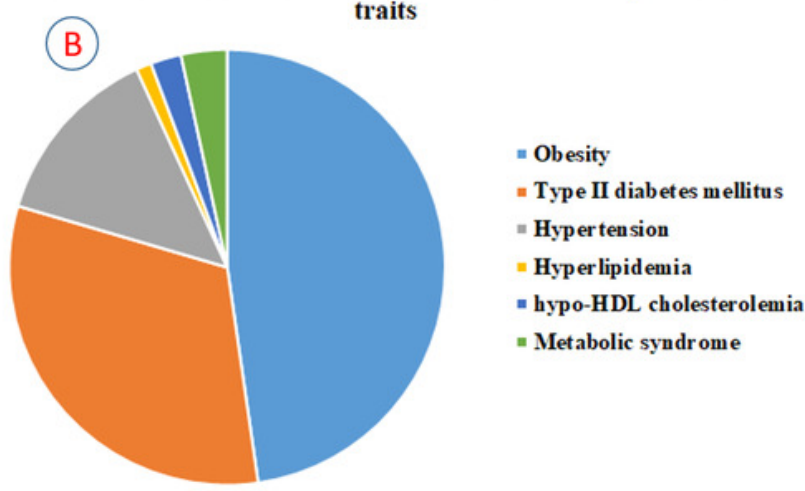

The distribution of IncRNAs in different species

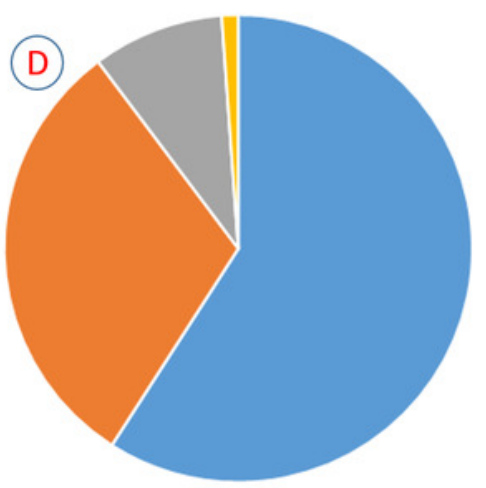

Hom o sapiens

- Mus musculus

- Rattus norvegicus

- Sus scrofa 


\section{Figure 3}

The schematic workflow of the ncRNA2MetS database

(A) Browse the data. (B) Search the data. (C) Browse the query results. (D) Browse the detail information about a specific MetS-ncRNA association. (E) Submit a new MetS-ncRNA association to the ncRNA2MetS database.

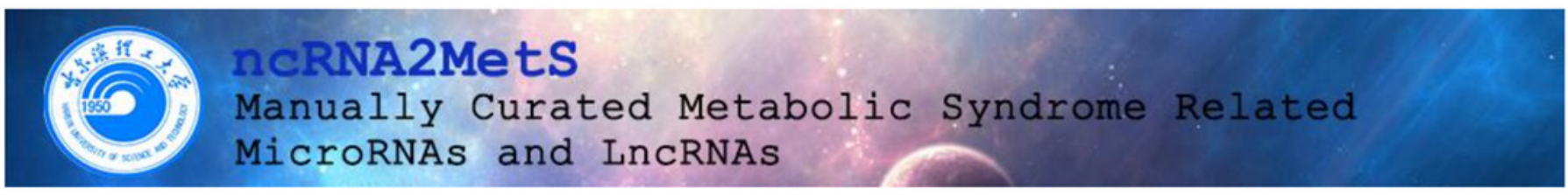

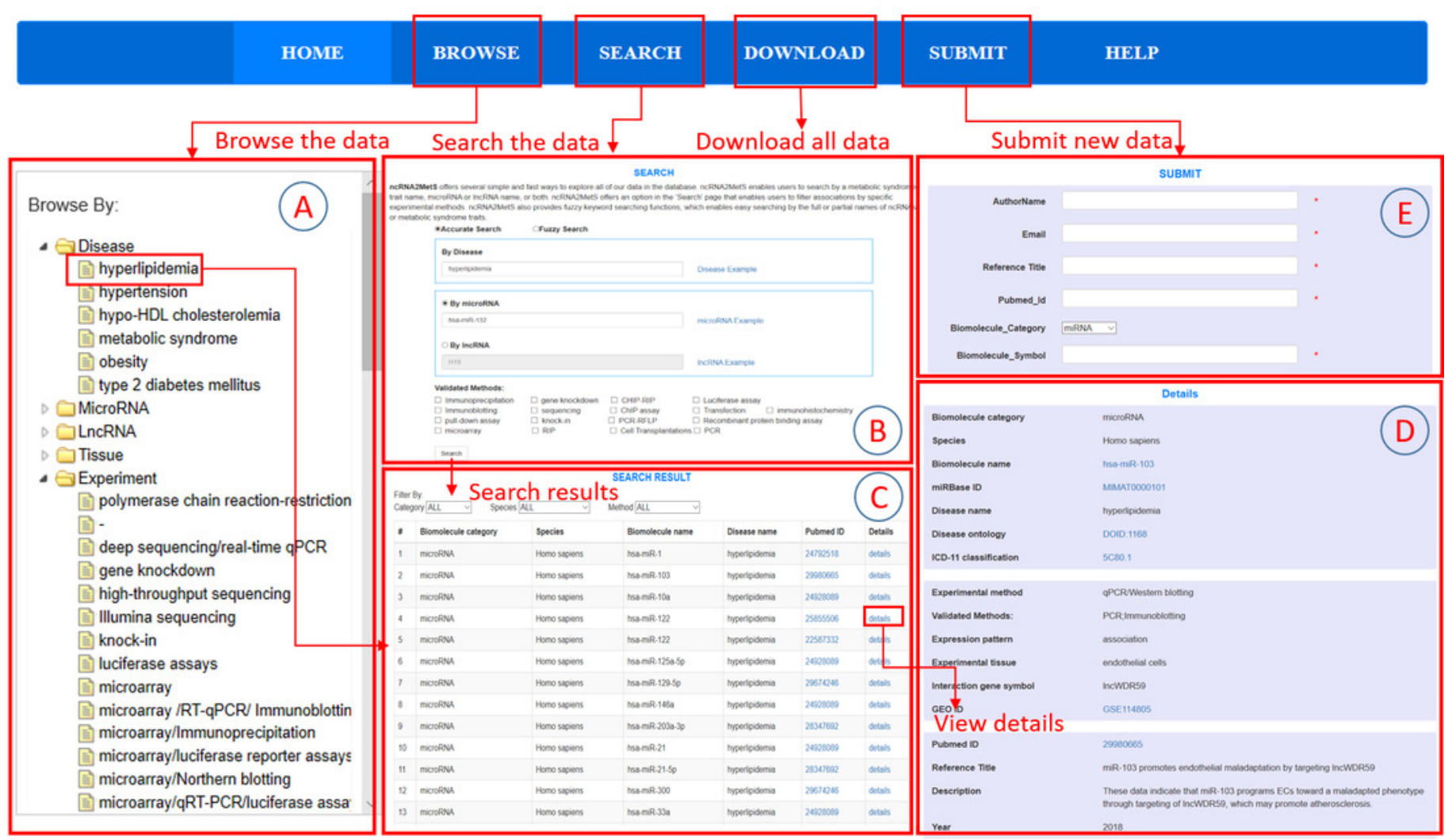


Figure 4

The MetS-ncRNA association network

Nodes correspond to ncRNAs (miRNAs and IncRNAs) and metabolic syndrome traits (central obesity, type 2 diabetes mellitus, hypertension, hyperlipidaemia and hypo-HDL cholesterolemia) and the edges correspond to experimentally supported associations. The size of the nodes corresponds to the nodes' degree.

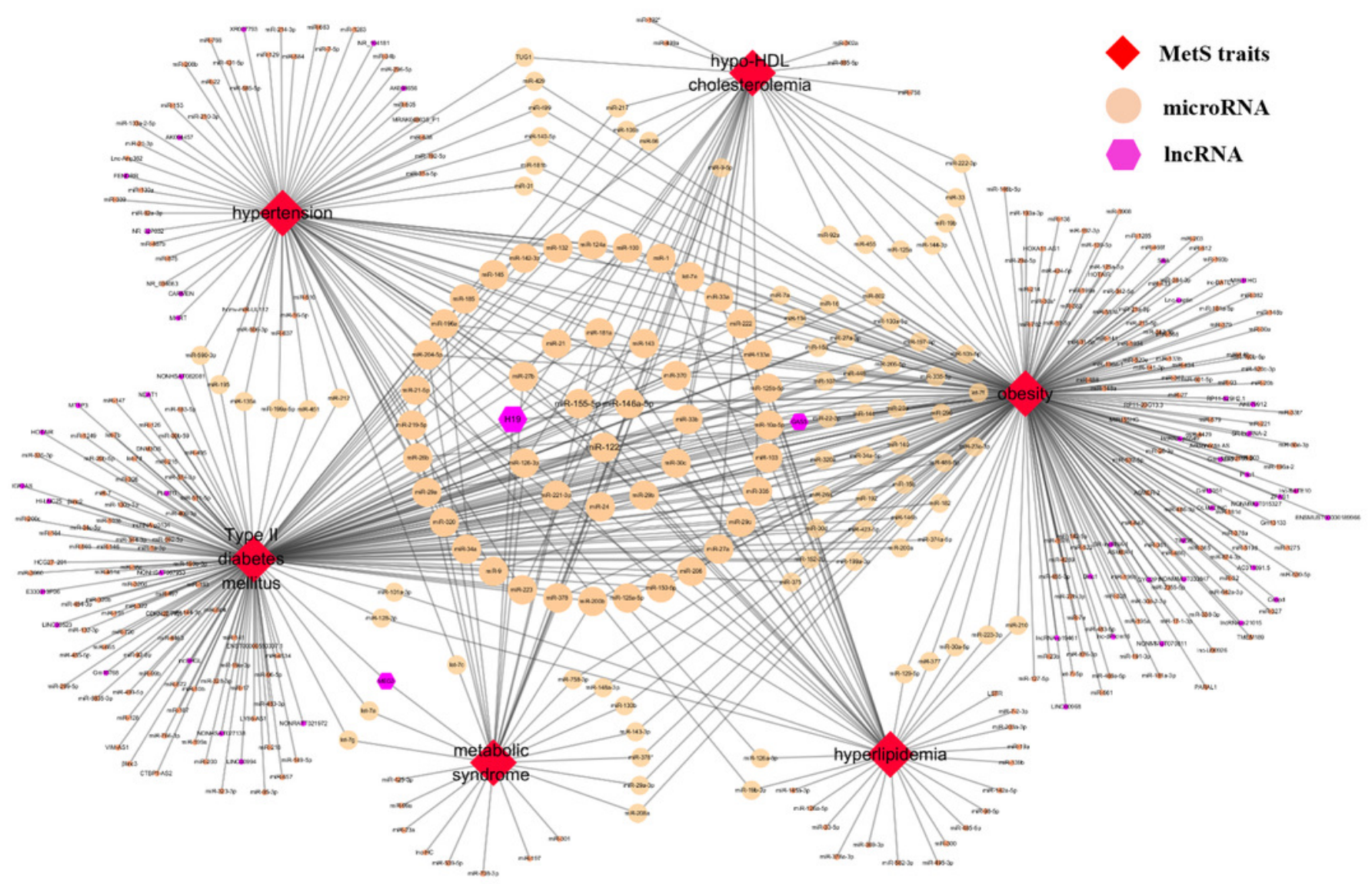


Figure 5

Comparison of the number of ncRNAs associated with metabolic syndrome in different databases

(A) Comparison of the number of ncRNAs associated with metabolic syndrome among

BioM2MetDisease, HMDD and ncRNA2MetS. (B) Number of papers about ncRNAs associated with metabolic syndrome between 2007 and April 2019.
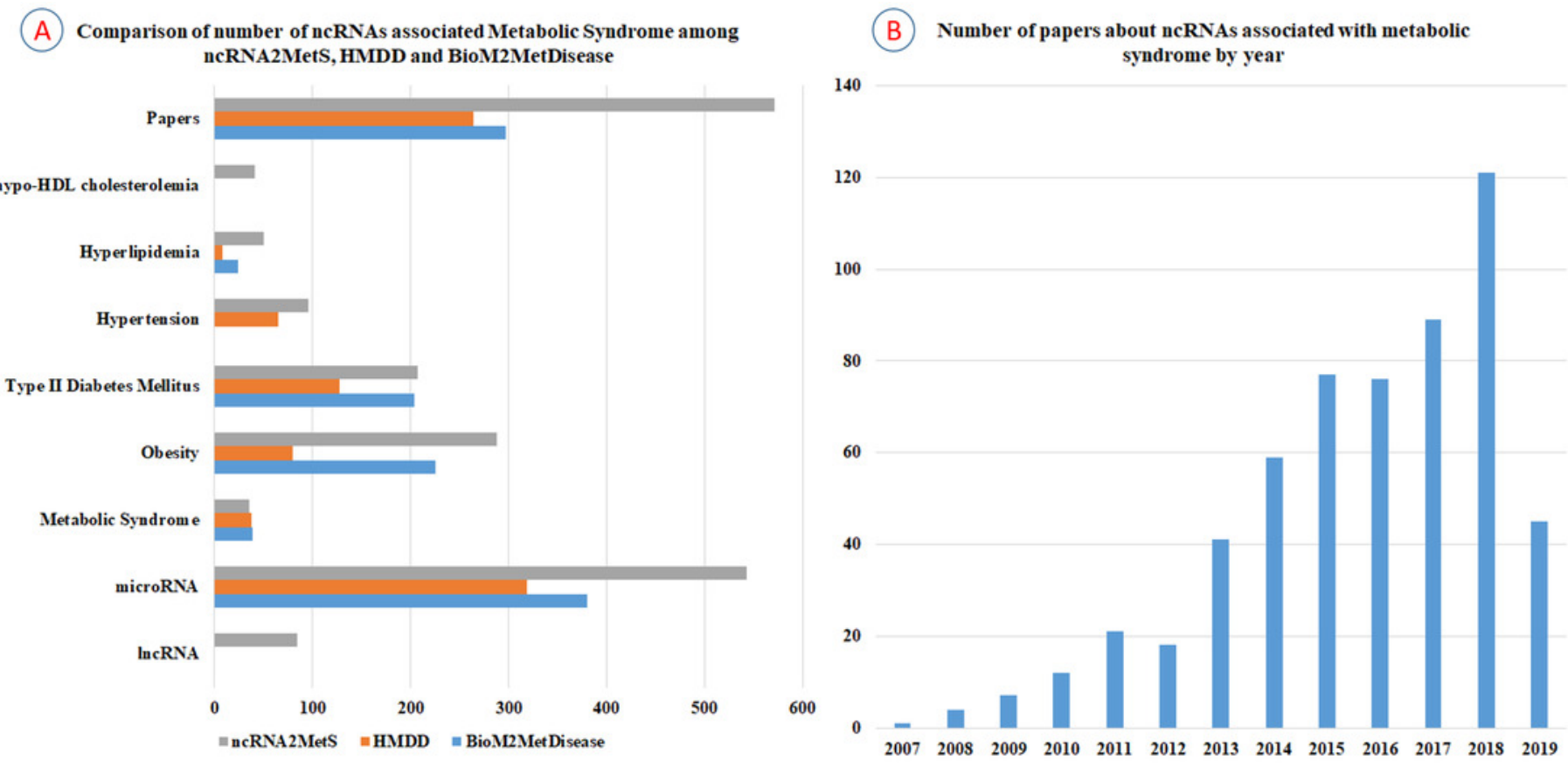